\title{
Uso da chupeta como fator preditivo a interrupção precoce da amamentação: Uma revisão integrativa
}

\author{
Use of the pacifier as a predictive factor for early breastfeeding: An integrative review \\ Uso del chupete como factor predictivo para la lactancia temprana: Una revisión integrativa
}

Recebido: 31/05/2021 | Revisado: 08/06/2021 | Aceito: 21/06/2021 | Publicado: 04/07/2021

Sheron Maria Silva Santos

ORCID: https://orcid.org/0000-0002-7492-3604 Universidade Regional do Cariri, Brasil

E-mail: sheronmss@hotmail.com

Andressa de Sá Barreto Dantas

ORCID: https://orcid.org/0000-0001-8402-295X Universidade Regional do Cariri, Brasil

E-mail: andressadesa19@hotmail.com

Edilma Gomes Rocha Cavalcante

ORCID: https://orcid.org/0000-0002-6861-2383 Universidade Regional do Cariri, Brasil

E-mail: edilma.gomes@urca.br

Alissan Karine Lima Martins

ORCID: https://orcid.org/0000-0002-4203-6656 Universidade Regional do Cariri, Brasi

E-mail: alissan.martins@urca.br

Evanira Rodrigues Maia

ORCID: https://orcid.org/0000-0001-9377-7430 Universidade Regional do Cariri, Brasil

E-mail: evanira.maia@urca.br

Grayce Alencar Albuquerque

ORCID: https://orcid.org/0000-0002-8726-0619 Universidade Regional do Cariri, Brasil E-mail: grayce.alencar@urca.br

Maria do Socorro Vieira Lopes

ORCID: https://orcid.org/0000-0003-1335-5487 Universidade Regional do Cariri, Brasil E-mail: socorro.lopes@urca.br

Inês Dolores Teles Figueiredo

ORCID: https://orcid.org/0000-0002-7280-8442 Universidade Regional do Cariri, Brasil E-mail: ines.teles@urca.br

Felice Teles Lira dos Santos Moreira

ORCID: https://orcid.org/0000-0002-1979-5232 Universidade Regional do Cariri, Brasil E-mail: felice.teles@urca.br

\section{Resumo}

A pesquisa objetivou identificar a relação do uso da chupeta com a interrupção precoce da amamentação por meio dos achados da literatura científica. Trata-se de uma revisão integrativa realizada em março de 2020 através de achados na Biblioteca Virtual em Saúde, PubMed/Medline e Scientific Eletronic Library Online com os descritores em saúde: Aleitamento Materno/Breast Feeding, chupetas/Pacifiers; e a palavra-chave irregular: interrupção da amamentação/interruption of breast feeding, utilizando o operador booleano and para o cruzamento dos termos utilizados. A busca por achados na literatura resultou em seis artigos. Evidenciou-se que algumas mães utilizam a chupeta como objeto de consolo ao seu bebê logo nos seus primeiros meses de vida e que essa prática tem contribuído com a interrupção precoce da amamentação devido ao formato do bico da chupeta ser inversamente proporcional ao do mamilo, aspecto que faz o bebê rejeitá-lo, uma vez que sugar o mamilo é uma tarefa árdua e cansativa, ao contrário do que se é realizado com a chupeta. Neste sentido, o uso da chupeta é apontado como um fator preditivo a interrupção precoce da amamentação fazendo-se necessário intensificar a prática de estratégias educativas que visem esclarecer e orientar sobre os riscos do uso da chupeta durante o aleitamento materno.

Palavras-chave: Aleitamento materno; Chupetas; Fator de risco. 


\begin{abstract}
The research aimed to identify the relationship between the use of a pacifier and the early interruption of breastfeeding through the findings of the scientific literature. This is an integrative review carried out in March 2020 through findings in the Virtual Health Library, PubMed / Medline and Scientific Eletronic Library Online with the health descriptors: Breast Feeding / Breast Feeding, pacifiers / Pacifiers; and the irregular keyword: interruption of breastfeeding / interruption of breast feeding, using the Boolean operator and for the crossing of the terms used. The search for findings in the literature resulted in six articles. It was evidenced that some mothers use the pacifier as an object of comfort to their baby in their first months of life and that this practice has contributed to the early interruption of breastfeeding due to the shape of the pacifier tip being inversely proportional to that of the nipple, an aspect which makes the baby reject it, since sucking on the nipple is an arduous and tiring task, unlike what is done with a pacifier. In this sense, the use of the pacifier is pointed out as a predictive factor for the early interruption of breastfeeding, making it necessary to intensify the practice of educational strategies that aim to clarify and guide about the risks of using a pacifier during breastfeeding.
\end{abstract}

Keywords: Breast feeding; Pacifiers; Risk factors.

\title{
Resumen
}

La investigación tuvo como objetivo identificar la relación entre el uso de un chupete y la interrupción temprana de la lactancia materna a través de los hallazgos de la literatura científica. Se trata de una revisión integradora realizada en marzo de 2020 a través de hallazgos en la Biblioteca Virtual de Salud, PubMed / Medline y Scientific Eletronic Library Online con los descriptores de salud: Lactancia materna / Lactancia materna, chupetes / Chupetes; y la palabra clave irregular: interrupción de la lactancia materna / interrupción de la lactancia materna, utilizando el operador booleano y por el cruce de los términos utilizados. La búsqueda de hallazgos en la literatura resultó en seis artículos. Se evidenció que algunas madres utilizan el chupete como objeto de consuelo para su bebé en sus primeros meses de vida y que esta práctica ha contribuido a la interrupción precoz de la lactancia materna debido a que la forma de la punta del chupete es inversamente proporcional a la del chupete. El pezón, aspecto que hace que el bebé lo rechace, ya que succionar el pezón es una tarea ardua y agotadora, a diferencia de lo que se hace con un chupete. En este sentido, se señala el uso del chupete como factor predictivo de la interrupción precoz de la lactancia materna, por lo que es necesario intensificar la práctica de estrategias educativas que tengan como objetivo esclarecer y orientar sobre los riesgos del uso del chupete durante la lactancia.

Palabras clave: Lactancia materna; Chupetes; Factores de riesgo.

\section{Introdução}

A amamentação, também mencionada como aleitamento materno, diz respeito ao método de nutrição de maior eficácia e eficiência para os primeiros meses de vida da criança por ser natural, contemplar todos os nutrientes necessários ao organismo do bebê, anticorpos e água, além de ser, econômico, prático e rápido (Brasil 2015; Margotti \& Margotti 2017).

Os benefícios da amamentação são muitos e estão relacionados tanto aos aspectos nutricionais como emocionais. Além de ser uma fonte de nutrientes, promove uma interação entre o binômio mãe-bebê, desenvolvendo laços afetivos, segurança e acolhimento (Andrade, 2014).

O aleitamento materno pode ser exclusivo, predominante, complementado, misto ou parcial. Dentre estes, destaca-se o Aleitamento Materno Exclusivo (AME), pois se trata da amamentação apenas com leite materno, ofertado através da mama ou ordenhado, sem a presença de outra fonte de alimentação liquida ou sólida (Campos et al., 2015).

Além disso, é através da amamentação que a criança irá desenvolver a formação correta da relação maxilo-mandibular e proporcionará o crescimento correto da região orofacial (Gisfrede et al., 2016).

A Organização Mundial de Saúde (OMS) orienta o AME nos primeiros seis meses de vida da criança e de forma complementar até os dois anos de vida ou mais (Brasil 2015, p. 15). Todavia, segundo dados do Fundo das Nações Unidas para a Infância (UNICEF, 2013) verifica-se que apenas 40\% das mulheres realizam o AME aos seus bebês (Organização das Nações Unidas, Brasil - ONUBR, 2017).

Esse fenômeno pode ser justificado devido a amamentação ser um processo complicado de ser realizado, exigir esforços da mulher e poder vir acompanhado de fatores que podem dificultar sua execução como, por exemplo: crença sobre leite fraco, mastalgia, ductos lactíferos bloqueados, ingurgitamento mamário, mastite, absesso mamário, fissuras mamilares, pega incorreta e o tempo de licença maternidade (Freitas, Werneck \& Borim, 2018). 
Por essa razão, esses fatores podem culminar o desmame precoce da amamentação antes dos seis meses de vida e a introdução de outros alimentos na dieta da criança que estava em aleitamento materno exclusivo (Prado, Fabbro \& Ferreira, 2016).

Acredita-se também que a chupeta pode ser um fator contribuinte com a interrupção da amamentação, pois, a partir do seu uso, a criança posiciona a língua sobre o seio materno no ato da amamentação, não conseguindo sugar o leite e, consequentemente, rejeita-o, (Gisfrede et al, 2016). Além disso, as mães que possuem pouca motivação para amamentar, muitas vezes, acabam introduzindo a chupeta como uma forma de consolar a criança (Andrade, 2014).

Dessa forma, questiona-se: O uso da chupeta está relacionado com a interrupção precoce da amamentação?

O presente estudo tem como objetivo identificar a relação do uso da chupeta com a interrupção precoce da amamentação por meio dos achados da literatura científica.

\section{Metodologia}

A presente pesquisa possui abordagem qualitativa, cunho exploratório e se trata de uma revisão integrativa da literatura que, segundo Silveira, Mendes e Galvão (2019), diz respeito ao estudo que facilita o entendimento e compreensão sobre determinado conteúdo a partir da síntese de pesquisas científicas.

Dessa forma, o estudo foi fundamentado em seis etapas: (1) elaboração da pergunta norteadora; (2) busca e seleção dos estudos; (3) extração de dados; (4) avaliação dos estudos inclusos na revisão; (5) síntese dos resultados; (6) e apresentação da revisão.

A pergunta norteadora e os descritores em saúde foram contemplados pela estratégia PVO, em que "P" refere-se ao problema da pesquisa = interrupção da amamentação; "V" às variáveis do estudo = uso da chupeta; e "O" o desfecho esperado = relação do uso da chupeta com a interrupção precoce da amamentação.

A coleta de dados foi realizada no mês de março de 2020 de forma pareada com dois pesquisadores, para maior crivo metodológico e confiabilidade da seleção dos estudos, por meio do acervo publico em formato de artigo científico disponibilizado nas bases de dados Biblioteca Virtual em Saúde (BVS), PubMed/Medline e Scientific Eletronic Library Online (SciELO).

A busca dos artigos foi realizada através da alocação dos descritores em saúde (DeCS/MeSH), a saber: "Aleitamento Materno"/"Breast Feeding", chupetas/Pacifiers; e a palavra-chave irregular: "interrupção da amamentação"/”interruption of breastfeeding", nas bases de dados selecionadas, sendo os descritores em português na BVS e SciELO e os em inglês na PubMed/Medline.

Devido à escassez dos resultados encontrados com os descritores, foi decidido utilizar uma palavra-chave irregular (interrupção da amamentação) devido o cruzamento com outros descritores conferir resultados escassos em todos os bancos de dados. Ademais, seu uso proporcionou achados que melhor se adequava ao objetivo proposto na pesquisa.

É importante mencionar que o cruzamento dos descritores foi realizado por meio do operador booleano and com intuito de correlacionar os termos utilizados para alcançar um resultado mais próximo do objetivo.

Para a seleção dos artigos disponibilizados nas bases de dados foram traçados os critérios de inclusão e exclusão, a saber: (a) inclusão: artigos disponibilizados no formato completo escritos na língua portuguesa ou inglesa nos últimos dez anos (2010- 2020); (b) exclusão: artigos repetidos, duplicados, não disponíveis no formato gratuito e que em seu título ou resumo não possuíam relação com a temática em discussão.

Neste sentido, os artigos que se adequaram aos critérios estabelecidos, foram analisados por meio da leitura crítica e reflexiva das pesquisas, interpretando seus principais achados e discutindo as ideias dos autores, confrontando-as ou corroborando-as, tentando responder a questão que norteia o presente estudo. 
Dessa forma, os dados foram organizados por meio de quadros, construídos com auxílio do programa Microsoft Office, o Excel 2017, expondo e comparando as características das publicações como autoria, período de publicação do estudo, lócus do estudo, objetivo e principais resultados. Após identificação desses pontos, os dados foram analisados criticamente conforme a literatura revisada.

\section{Resultados e Discussão}

$\mathrm{O}$ cruzamento dos descritores Aleitamento Materno/Breast Feeding, chupetas/Pacifers e a palavra-chave irregular: interrupção da amamentação/interruption of breastfeeding na BVS, PubMed/Medline e SciELO resultou em vinte e nove documentos, dos quais após aplicação dos critérios de inclusão e exclusão pré-estabelecidos permaneceram seis artigos. O caminho percorrido para seleção dos estudos, pode ser visualizado no Fluxograma 1.

Fluxograma 1: Caminho percorrido para seleção dos artigos.

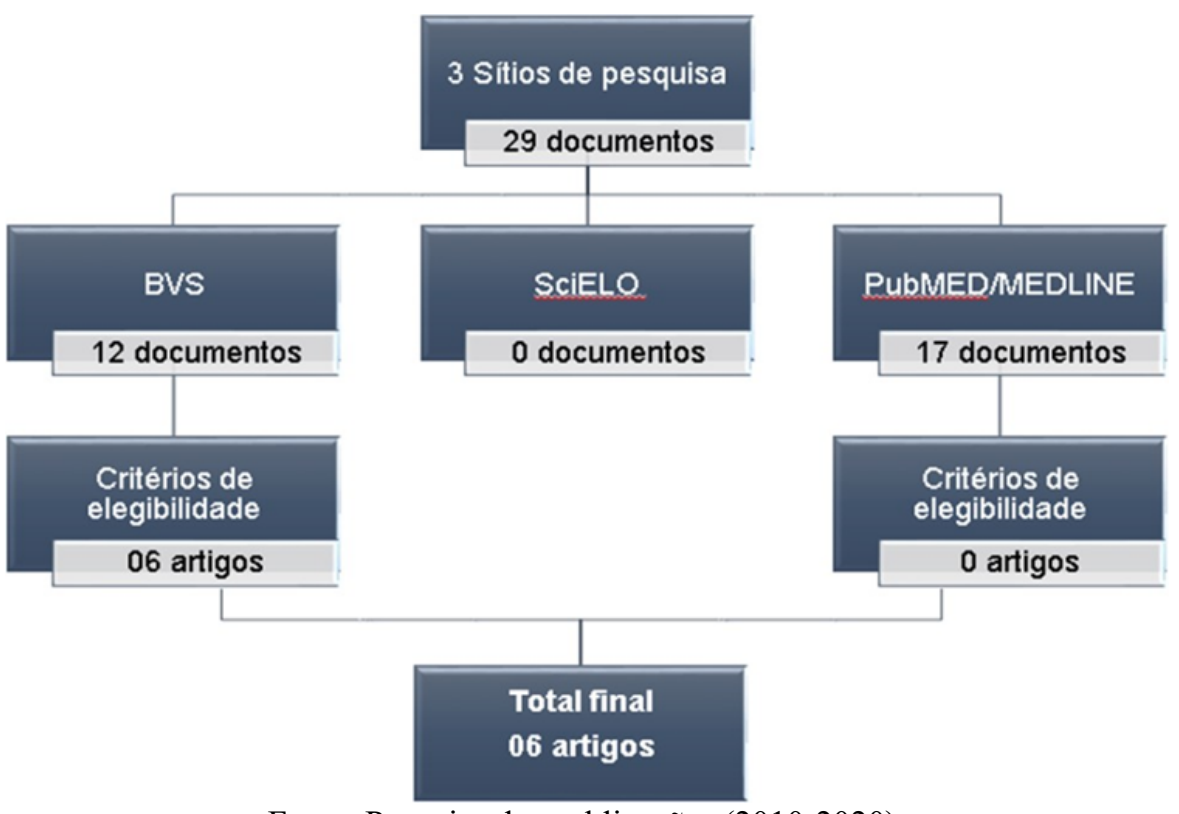

Fonte: Pesquisa das publicações (2010-2020).

O Fluxograma 1, chama atenção para os achados encontrados na base de dados PubMED, uma vez que após aplicação dos critérios de inclusão e exclusão, nenhum documento se adequou aos quesitos estabelecidos; resultado semelhante ao apresentado na SciELO, tendo em vista a alocação dos descritores em seu sítio de busca não proporcionar nenhum resultado, fazendo com que $100 \%(\mathrm{~N}=6)$ dos artigos utilizados para a construção dessa revisão fossem somente vinculados a BVS.

Este achado demonstra carência de pesquisas relacionadas à temática em discussão, devido ao baixo quantitativo de artigos disponíveis nas bases de dados, mesmo em um intervalo de tempo extenso (10 anos), fortalecendo a importância de sua realização tanto no âmbito social quanto no meio acadêmico, tendo em vista tratar-se diretamente da saúde da criança e envolver aspectos relacionados aos objetivos de desenvolvimento sustentável como, por exemplo, a boa saúde e o bem-estar (ONUBR, 2015).

Constatou-se predominância do idioma português, tipo de estudo de intervenção e os profissionais da área de odontologia e enfermagem são os que mais pesquisam sobre a relação do uso da chupeta com a interrupção precoce da amamentação. Com isso, é fundamental que ocorra mais engajamento de outras áreas, visando gerar novas descobertas e discussões, tanto em outros idiomas, como também em outros tipos de estudo.

Ainda sobre a caracterização dos artigos selecionados, construiu-se o Quadro 1, para apreciação e melhor visualização 
sobre as variáveis envolvendo autor(es), período do estudo, lócus do estudo, objetivo e principal resultado.

Quadro 1: Caracterização das variáveis dos artigos.

\begin{tabular}{|c|c|c|c|}
\hline $\begin{array}{c}\text { AUTOR/ } \\
\text { ANO }\end{array}$ & $\begin{array}{c}\text { LÓCUS DO } \\
\text { ESTUDO }\end{array}$ & OBJETIVO & PRINCIPAL RESULTADO \\
\hline $\begin{array}{l}\text { Vieira et } \\
\text { al. (2010) }\end{array}$ & $\begin{array}{l}\text { Maternidades } \\
\text { do município de } \\
\text { Feira de } \\
\text { Santana. }\end{array}$ & $\begin{array}{l}\text { Averiguar os fatores associados } \\
\text { à interrupção do aleitamento } \\
\text { materno exclusivo no primeiro } \\
\text { mês de lactação na cidade de } \\
\text { Feira de Santana (BA). }\end{array}$ & $\begin{array}{l}\text { Falta de experiência prévia com amamentação (razão } \\
\text { de prevalência } 1,24 \text {; IC95\% } 1,75-1,43 \text { ), presença de } \\
\text { fissura mamilar (razão de prevalência } 1,25 \text {; IC } 95 \% \\
1,09-1,43 \text { ), horários pré- determinados para } \\
\text { amamentar (razão de prevalência } 1,42 \text {; IC95\% } 1,09- \\
1,84 \text { ) e uso de chupeta (razão de prevalência } 1,53 \text {; } \\
\text { IC95\% } 1,34-1,76 \text { ) foram } \\
\text { identificados como fatores preditivos da interrupção } \\
\text { do aleitamento exclusivo. }\end{array}$ \\
\hline $\begin{array}{l}\text { Sanches } \\
\text { et al. } \\
(2011)\end{array}$ & $\begin{array}{l}\text { Unidades } \\
\text { básicas de saúde } \\
\text { (UBS) } \\
\text { periferia da } \\
\text { Município do } \\
\text { São Paulo }\end{array}$ & $\begin{array}{l}\text { Identificar os fatores associados } \\
\text { à interrupção do aleitamento } \\
\text { materno exclusivo (AME) de } \\
\text { lactentes nascidos com baixo } \\
\text { peso assistidos na Atenção } \\
\text { Básica }\end{array}$ & $\begin{array}{l}\text { Identificou-se associado à interrupção do AME no } \\
\text { terceiro mês: idade materna } \\
<18 \text { anos; vínculo empregatício informal (como fator } \\
\text { de proteção); ingestão de álcool na gestação; < } 6 \\
\text { consultas no pré-natal; gestação múltipla; peso ao } \\
\text { nascer } \leq 2.000 \mathrm{~g} \text {; dificuldade na primeira mamada; } \\
\text { queixa sobre a amamentação no primeiro mês; uso de } \\
\text { chupeta no primeiro e segundo meses. }\end{array}$ \\
\hline $\begin{array}{l}\text { Queluz et } \\
\text { al. (2011) }\end{array}$ & Serrana-SP & $\begin{array}{l}\text { Identificar a prevalência e os } \\
\text { determinantes do aleitamento } \\
\text { materno exclusivo em crianças } \\
\text { menores de } 6 \text { meses, no } \\
\text { município de Serrana-SP }\end{array}$ & $\begin{array}{l}\text { Nas análises univariadas, verificou-se que mães que } \\
\text { trabalham fora sem licença-maternidade, mães que } \\
\text { não trabalham fora, adolescentes e o uso de chupeta } \\
\text { apresentaram maior chance de interrupção do } \\
\text { aleitamento materno exclusivo. Na análise } \\
\text { multivariada, as mães que trabalham fora sem licença- } \\
\text { maternidade têm } 3 \text { vezes mais chance de } \\
\text { desmamarem precocemente seus filhos. }\end{array}$ \\
\hline $\begin{array}{l}\text { Warkentin } \\
\text { et al. } \\
(2012)\end{array}$ & $\begin{array}{l}\text { Creches } \\
\text { públicas e } \\
\text { filantrópicado } \\
\text { Município de } \\
\text { São Paulo }\end{array}$ & $\begin{array}{l}\text { Descrever as prevalências de } \\
\text { aleitamento materno exclusivo e } \\
\text { avaliar os deter- minantes } \\
\text { associados à sua duração entre } \\
\text { crianças frequentadoras de } \\
\text { creches públicas e filantrópicas } \\
\text { do Município de São Paulo. }\end{array}$ & $\begin{array}{l}\text { O tempo médio de aleitamento materno exclusivo foi } \\
\text { de } 87,2 \pm 61,36 \text {, mediana de } 90 \text { dias. Na análise } \\
\text { multivariada, en- traram para o modelo final as } \\
\text { seguintes variáveis: idade da mãe }<20 \text { anos } \\
(\mathrm{HR}=1,43,95 \% \mathrm{CI}=1,09-1,88) \text {, uso de } \\
\text { chupeta com }<3 \text { meses }(\mathrm{HR}=1,87,95 \% \mathrm{CI}=1,57- \\
2,24) \text { e prematuridade }(\mathrm{HR}=1,36,95 \% \mathrm{CI}=1,04-1,77) \text {. }\end{array}$ \\
\hline $\begin{array}{l}\text { Uema et } \\
\text { al. (2015) }\end{array}$ & $\begin{array}{l}\text { Bases de dados } \\
\text { (Lilacs, Scielo, } \\
\text { Cochrane, } \\
\text { Bdenf, Medline } \\
\text { e PubMed). }\end{array}$ & $\begin{array}{l}\text { Comparar as prevalências e os } \\
\text { fatores determinantes do } \\
\text { aleitamento materno em } \\
\text { diferentes cidades e estados } \\
\text { brasileiros, a partir de pesquisas } \\
\text { que utilizaram metodologia } \\
\text { semelhante à proposta pelo } \\
\text { Projeto Amamentação e } \\
\text { Municípios }\end{array}$ & $\begin{array}{l}\text { Algumas variáveis maternas são apontadas como } \\
\text { possíveis responsáveis pelo desmame precoce, tais } \\
\text { como baixa renda e baixa escolaridade. No que tange } \\
\text { às variáveis do lactente, a chupeta aparece como o } \\
\text { maior fator agravante para o desmame. }\end{array}$ \\
\hline $\begin{array}{l}\text { Buccini } \\
\text { (2017) }\end{array}$ & $\begin{array}{l}\text { Bases de dados } \\
\text { (CINAHL, } \\
\text { Scopus, Web of } \\
\text { Science, } \\
\text { LILACS, } \\
\text { Medline) }\end{array}$ & $\begin{array}{l}\text { Analisar a associação entre uso } \\
\text { de chupeta e interrupção do } \\
\text { AME, bem como a evolução do } \\
\text { uso de chupeta e sua influência } \\
\text { no AME em menores de } 6 \text { meses } \\
\text { nas Capitais Brasileiras e no DF, } \\
\text { 1999-2008. }\end{array}$ & $\begin{array}{l}\text { O uso de chupeta é o fator mais fortemente associado } \\
\text { à interrupção precoce do AME no Brasil no período } \\
\text { de } 1999 \text { a } 2008 \text { (OR ajustado= } 2,77 \text {; IC } 95 \%=2,63- \\
2,91) \text {. }\end{array}$ \\
\hline
\end{tabular}

Fonte: Pesquisa das publicações (2010-2020).

A partir do Quadro 1, percebe-se que no intervalo de tempo de 2010-2020 o quantitativo de estudos publicados a cada ano resumem-se entre nenhum, 1 (um) ou 2 (dois), com prevalência para a ausência de registros, principalmenteno que serefereaos últimos 5 (cinco) anos, deixando clara a carência de estudos sobre a associaçãodo uso da chupeta com a interrupção 
precoce da amamentação, principalmente escassez de pesquisas com periodicidade recente.

A frequência das pesquisas em 2010, 2011 e 2012 podem ser justificadas devido a publicação da Lei No 11.265 em3 de Janeiro de 2006 trazer em seus artigos,parágrafos e incisos como deve ser feita a comercialização de produtos alimentícios e de puericultura para lactentes e crianças de primeira infância, deixando claro em seus rótulos ou propagandas afins, informações sobre a importância da amamentaçãomaterna exclusiva até os seis meses de vida (Brasil, 2006).

No que tange ao local de realização dos estudos, compreende-se que há prevalência de documentos executados na região sudeste, especialmente no estado de São Paulo. Este fato mostra o quanto existe carência de pesquisas nas outras regiões, principalmente no nordeste, que apresenta menor prevalência de aleitamentomaterno exclusivo.

Sobre o principal objetivo dos autores com as pesquisas realizadas, foi verificado buscas semelhantes entre eles, aspecto evidenciado por meio da sinonímiae/ou similaridade dos verbos empregados em seus objetivos como, por exemplo: averiguar, identificar e analisar os fatores associados com a interrupção do aleitamento materno.

Sob essa ótica, os autores apresentaram resultados semelhantes, corroboraram entre si e apontaram outras causas, além das descritas no Art. $16 \S 1^{\circ}$ da Lei $\mathrm{N}^{\circ} 11.265$, o uso de mamadeira, bico ou chupeta, como fatores prejudiciais e preditivos ao abandono do aleitamento materno (Brasil, 2006).

De acordo com Vieira e colaboradores (2010), a chupeta é um dos principais fatores responsáveis pela interrupção precoce do Aleitamento Materno Exclusivo (AME), tendo em vista minimizar o quantitativo de mamadas diárias. Em detrimento disso, a mãe passa a ter uma menor estimulação na região mamilo-areolar, ocasionando a diminuição da produtividade do leite.

Em contrapartida, os estudos realizados com 275 crianças menores de seis meses, do município de Serrana-SP, apontaram que a chupeta não é o principal fator que provoca a interrupção do aleitamento materno. Este fenômeno, por sua vez, é atribuído ao distanciamento entre o binômio mãe-bebê em detrimento do exercício profissional da mulher, onde, seu direito a licença maternidade em algumas empresas é negligenciado e necessita retornar as atividades laborais de forma precoce (Queluz et al., 2011).

Contudo, embora Queluz e colaboradores (2011) não apontem a chupeta como o principal fator preditivo a da interrupção precoce da AME, os autores descrevem sobre a importância de orientar as nutrizes sobre a não oferta da chupeta aos seus bebês, uma vez que este utensílio também contribuir para a interrupção do AME e que o alívio para o choro do bebê deve feito com a oferta do próprio seio materno e da proximidade dos pais e da família com a criança.

Para os pesquisadores Sanches e colaboradores (2011), a chupeta está entre os fatores que provocam a interrupção do AME nos dois primeiros meses e salientam que seu uso passa a ser considerado um fator de risco para redução do AME.

Concretizando com os pensamentos supramencionados, Warkentin e colaboradores (2012) afirmam que, através de pesquisas literárias foi possível verificar que a utilização da chupeta encontra-se diretamente associada com a redução do tempo adequado do aleitamento materno, pois, foi visto que $79,8 \%$ das crianças fazem uso da chupeta antes dos três meses e que estas possuem 1,87 vezes maiores indícios de terem o desmame precoce da amamentação quando comparadas àquelas que não faziam uso do objeto.

Ainda conforme os autores, esses percentuais foram encontrados com mais evidências nas capitais brasileiras, contabilizando em média $60,3 \%$ casos de crianças que usavam chupeta em seu cotidiano.

Já nos estudos de metanálise investigativa desenvolvido por Uema e colaboradores (2015), foi possível assegurar que a redução do AME é uma consequência que está inteiramente influenciada e interligada com o uso da chupeta, haja vista seu uso ter sido apontado como principal fator na maioria das pesquisas analisadas, comprovando sua correlação com o desmame precoce.

Além disso, os autores enfatizaram que os bebês que eram amamentados e faziam uso de chupeta, mostravam-se ter, em média, 22 vezes maior probabilidade de serem desmamados do que àqueles que não a utilizavam. 
Em um estudo observacional, Buccini (2017) identificou uma associação entre o uso da chupeta e interrupção do AME. Para esse autor, o uso de chupeta pode e deve ser considerado um fator de risco para a interrupção do AME em crianças menores de seis meses, sendo então percebido como um forte determinante para que a ocorrência da interrupção precoce dessa prática no Brasil.

Desse modo, devido ao uso da chupeta ser caracterizado como um fator de risco modificável é necessário que a prevenção de sua prática seja vista como fator preponente ao AME.

\section{Considerações Finais}

Diante dos dados apresentados, nota-se que a chupeta é um fator preditivo para a interrupção do aleitamento materno, sendo esta apontada como uma das principais causas associada ao seu acontecimento e que seu uso está relacionado como um método de consolo para a criança.

Portanto, faz-se necessário desenvolver estratégias educativas que visem esclarecer e orientar homens, mulheres e toda a família do bebê sobre as consequências vinculadas ao uso da chupeta para a vida do bebê

Por fim, as limitações do estudo se referem a carência de artigos, principalmente, contemporâneos, que fragilizam as conclusões acerca da relação do uso da chupeta com a interrupção da amamentação, sendo fundamental que novas pesquisas sejam realizadas para fortalecer a literatura científica sobre o AME e, principalmente, contribuir com a população, demonstrando, cientificamente, a relação do uso da chupeta como um fator diretamente relacionado com a interrupção precoce da amamentação.

\section{Referências}

Almeida, J. M. de, Luz, S. de A. B., \& Ued, F. da V. (2015). Support of breastfeeding by health professionals: integrative review of theliterature. Revista paulista de pediatria, 33(3), 355-362.

Andrade, I. S. N. (2014). Aleitamento materno e seus benefícios:primeiro passo para a promoção saúde. Revista Brasileira em Promoção daSaúde, 27(2), 149150 .

Brasil. (2015). Ministério da Saúde. Secretaria de Atenção à Saúde. Departamento de Atenção Básica. Saúde da criança: aleitamento materno e alimentação complementar. Brasília: Ministério da Saúde. http://bvsms.saude.gov.br/bvs/publicacoes/saude_crianca_aleitamento_materno_ca b23.pdf.

Brasil. (2006). Lei $N^{o} 11.265$, de 3 de janeiro de 2006. Regulamenta a comercialização de alimentos para lactentes e crianças de primeira infância e também a de produtos de puericultura correlatos. Brasília, DF: Ministério da Saúde. http://www.planalto.gov.br/ccivil_03/_Ato2004-2006/2006/Lei/L11265.htm.

Buccini, G. S. (2017). Evolução do uso de chupeta e sua influência no aleitamento materno exclusivo no Brasil, 1999-2008. (Tese de Doutorado em Nutrição em Saúde Pública). Faculdade de Saúde Pública da Universidade de São Paulo, Brasil.

Campos, A. M. de S., et al. (2015). Exclusive breastfeeding practicesreported by mothers and the introduction of additional liquids. Revista latino- americana de enfermagem, 23(2), 283-290.

Freitas, M. G., Werneck, A. L., \& Borim, B. C. (2018). Aleitamento Materno Exclusivo:adesão e dificuldades. Revista Enfermagem, $12(9)$, $2301-7$.

Gil, A. C. (2018). Como elaborar projetos de pesquisa. (6a ed.), Atlas.

Gisfrede, T. F., et al. (2016). Hábitos bucais deletérios e suas consequências em Odontopediatria. Rev. bras. odontol., 73(2), 144-9.

Leite, F. T. (2008). Metodologia Cientifica. Ideias e Letras.

Margotti, E., \& Margotti, W. (2017). Fatores relacionados ao Aleitamento Materno Exclusivo em bebês nascidos em hospital amigo da criança em uma capital do Norte brasileiro. Saúde em Debate, 41(1), 860-871.

Mendes, K. D. S., Silveira, R. C. C. P., \& Gavão, C. M. (2019). Uso de gerenciador de referências bibliográficas na seleção dos estudos primários em revisão integrativa. São Paulo, Texto \& Contexto Enfermagem, 28(1), 4-13.

Organização das Nações Unidas Brasil (ONUBR). (2017). Apenas 40\% das crianças são alimentadas exclusivamente com leite materno nos 6 primeiros meses de vida. ONUBR:2017. https://nacoesunidas.org/apenas-40-das-criancas-sao-alimentadas-exclusivamente-com-leite-materno-nos-6-primeiros-meses-de-vida/.

ONUBR. (2015). Transformando Nosso Mundo: A Agenda 2030 para o Desenvolvimento Sustentável. ONUBR. https://nacoesunidas.org/pos2015/agenda2030/.

Prado, C. V. C., Fabbro, M. R. C., \& Ferreira,G. I. (2016). Early weaning from breastfeeding from mothers perspective: a dialogical approach. Texto \& Contexto- 
Research, Society and Development, v. 10, n. 8, e2710816672, 2021

(CC BY 4.0) | ISSN 2525-3409 | DOI: http://dx.doi.org/10.33448/rsd-v10i8.16672

Enfermagem, 25(2), e1580015.

Queluz, M. C., et al. (2012). Prevalência e determinantes do aleitamento materno exclusivono município de Serrana, São Paulo, Brasil. Rev. Esc. Enferm. USP, $46(3), 537-543$

Sanches, M. T. C., et al. (2011). Fatores associados à interrupção do aleitamento maternoexclusivo de lactentes nascidos com baixo peso assistidos na atenção básica. Cad.Saúde Pública, 27(5), 953-965.

Uema, R. T. B., et al. (2015). Prevalência e fatores associados ao aleitamento materno noBrasil entre os anos 1998 e 2013: revisão sistemática. Seminário: Ciências Biológicas e da Saúde, 36(1), 349-362.

Vieira, G. O., et al. (2010). Fatores preditivos da interrupção do aleitamento materno exclusivo no primeiro mês de lactação. J Pediatr, 86(5), 441-444.

Warkentin, S., Viana, K. J., Zapana, P. M., \& Taddei, J. A. A. C. (2012). Fatores associados à interrupção do aleitamento materno exclusivo. Nutrire: Rev. Soc. Bras. Alim. Nutr, 37(2), 105-117. 